\title{
Supporting STEM Education Through High Altitude Balloon Platform Development
}

\author{
Zsófia Bodó, Bence Dávid Góczán \\ Simonyi Karoly College for Advanced Studies \\ Budapest, Hungary \\ bodo.zsofia@simonyi.bme.hu, goczan.bence@simonyi.bme.hu
}

\begin{abstract}
Qualified engineers with good theoretical and hands-on experience are vital for a country's healthy space industry. However, if a country lacks of space and aerospace related higher education opportunities, developing a full university master or bachelor program requires high effort. Therefore smaller and local educational projects may play significant role in talent management and development.
\end{abstract}

The UPRA Project (Universal Platform for Robotics and Aerospace) is a student project with an aim to develop a reliable, widely configurable, low maintenance, high altitude balloon platform for university research groups. The project not only offers flight opportunities but also provides hands-on experience on platform development, payload integration and project management.

Students who join the project can learn the main principles of space- and near-spacecraft development. Working on different subsystems requires different skill-sets which students can improve with the help of experienced team members and mentors from the space industry. Since a spacecraft is a complex system, project members are needed to specialize in different fields of engineering and science. This is an opportunity for students to gain confidence and experience in their field of interest of their later professional career and also helps them to select the proper path of their academic progress.

UPRA Project also offers flight opportunities for third-party payloads which require wider project management skills than a typical development project. To maintain a reliable launch service flight-planning, logistics, legal paperwork and field work at the launch event have to be done. All these activities are performed and organized by team members which increase their skills in project planning, project management and account management.

Beside university students the project also aims for the younger generation to promote STEM fields and reach out for the next generation of engineers. This goal let the team cooperate with 'Kids University' an event of Budapest University of Technology and Economics and also with the Space Camp of the Hungarian Astronautical Society. These partnerships made it possible to the project to demonstrate balloon launches to more than 250 young students in the first quarter of 2019.

Unmanned aerial vehicles and high altitude balloons are great assets of space education as they provide hands on experience through exciting engineering tasks which could be the base of the professional career of any student that takes part in the project.

Keywords-high altitude balloon, higher education, system engineering, STEM education

\section{INTRODUCTION}

One of the most important aspects of the project's educational motives is its flexible project roles. The team members have the opportunity to define learning goals for the upcoming semester. This involves the usage of flexible project roles. Thus the participants have the chance to apply to different roles and responsibilities to each and every launch event if these roles align with their skill development plan.

Besides focusing on engineering students who take part in the platform development project it is important to open towards other fields of space exploration. Providing launch opportunity to third party payloads allows non-engineering students to join the exploration of space by working on experiments that can be launched to the stratosphere.

This paper covers how the different parts of this high altitude balloon project can interact with students, which skills can they improve by selecting the given task and also introduces how the development of each subsystem of the balloon platform can enhance the aerospace engineering knowledge of a participant.

\section{UPRA BALLON PLATFORM}

The goal of the Universal Platform for Robotics and Aerospace (UPRA) Team of Simonyi Karoly College for Advanced Studies is to develop a configurable modular balloon platform for the aid of university research groups who are interested in sending payload to the stratosphere. The platform is designed and built completely by students and is able to fly small science payloads up to 30 kilometers altitude. The UPRA Team is planning to establish a launch service targeting university research groups to provide an affordable platform, launch, recovery and flight planning services for their research.

The platform provides a bidirectional radio communication, live position tracking, data collection and storage and science data transmission. The platform is equipped with redundant tracking and communication system and an independent landing tracker to narrow the landing area. 
The platform is highly configurable and requires low maintenance between flights which makes it suitable for frequent or tight schedule stratospheric missions. A standardized payload gondola is under development to lower the expenses and the payload-platform integration time.

Fig. 1 shows the exploded CAD illustration of the platformavionics module.

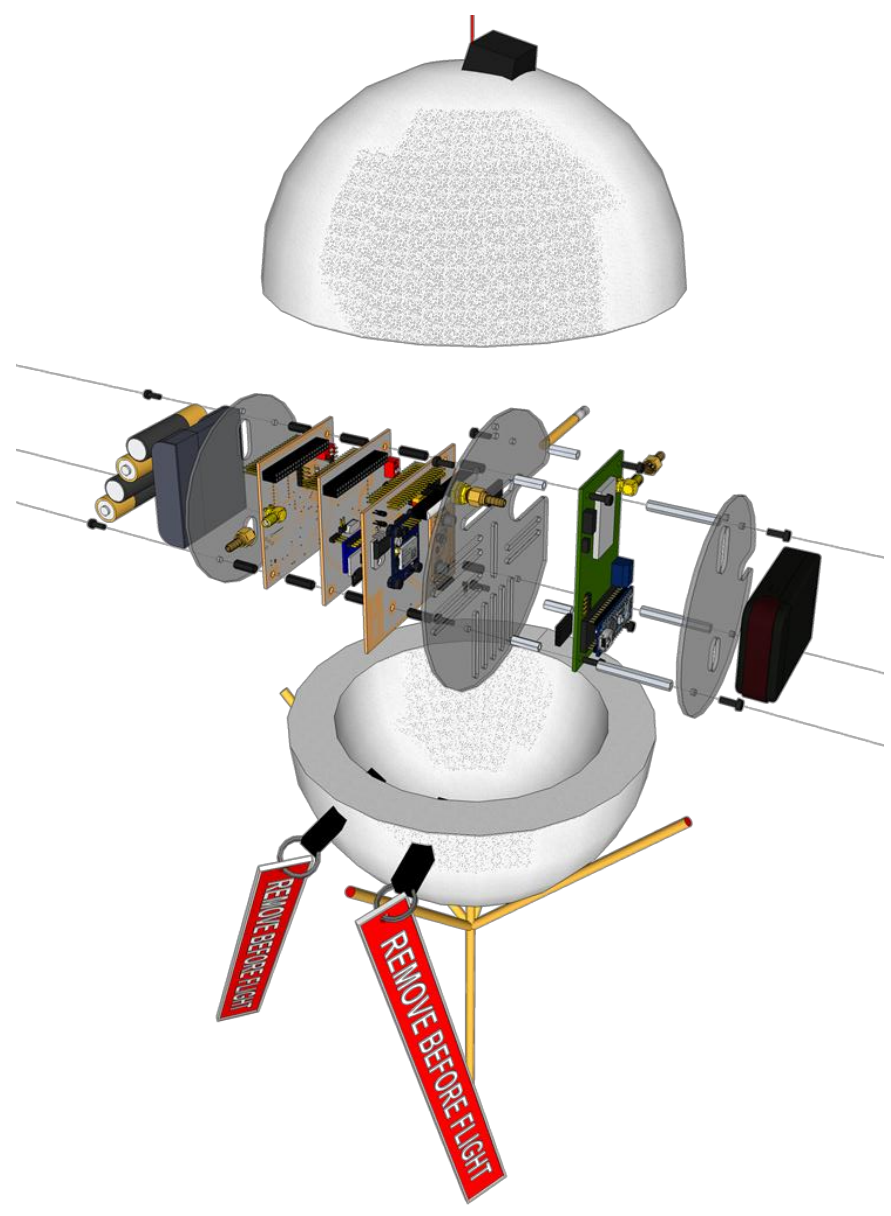

Fig. 1. UPRA Platform exploded view [4]

\section{Project Management}

The summer semester held two big opportunities for the students to take up in project manager role. We launched our Fly With Us campaign with the objective to support third-party organizations to develop payloads for stratospheric flights with our platform system and our launch and recovery service.

In July we took part in the Space Camp of the Hungarian Astronautical Society in the city of Sátoraljaújhely, Hungary. This annual camp is for high school students with interest in space and space related activities. Campers can take part in workshops, short seminars and trips to nearby museums. Every year guest presenters give some interesting lectures and organize hands-on activities that bring the campers closer to space exploration. In the Space Camp our Fly With Us programme had its first proof-of-concept flight. We had external payload, as requested by the organizers. The payload contained the official flag of Sátoraljaújhely, the host town of the event and presents to the participants. Since part of the payload was prepared as a camp activity the previous day, we had to present a quick and safe payload integration.

After the Space Camp we have been contacted by one of Hungary's most popular radio stations (Radio 1) to plan and conduct a stratospheric balloon flight for them (Fig. 2). Their request was to develop a payload, that represents one of Hungary's national symbols. This provided an extremely useful learning opportunity to the project manager of the launch, since the flight development had a challenging payload (lángos, a national food of Hungary), a tight development schedule and the flight was live-streamed and broadcasted to more than 100.000 people nationwide.

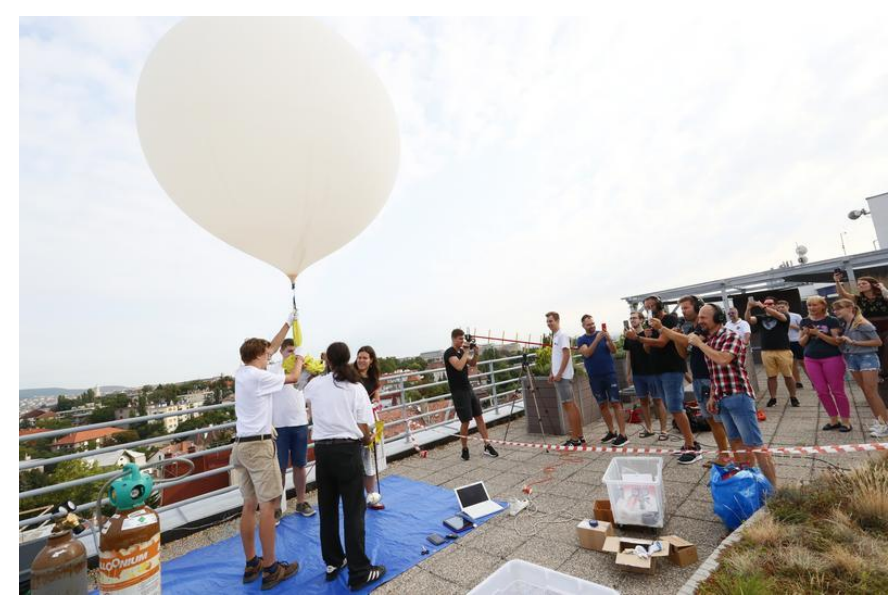

Fig. 2. Launch at Radio 1 headquarters as part of our Fly With Us programme [1]

\section{ON-BOARD COMPUTER (OBC)}

The On-Board Computer (OBC) is responsible for on-board data handling, collecting housekeeping data from the subsystems and controls the payload if necessary. Students working on the $\mathrm{OBC}$ become familiar with the development of microcontroller based real-time embedded systems. Gaining experience in developing fault-tolerant software, handling digital electronics and different communication protocols are valuable asset in the aerospace industry. Working with the development team does not only provide learning opportunity to design embedded circuitry but also tutor students in the assembly, integration and validation (AIV) process.

Even though the balloon platform is built with commercial off the shelf (COTS) components it needs to operate in extreme space-like environment. This requires the students to learn how to test and validate both the hardware and software modules. To ensure the reliability of the system validational tests are ran after every new modification on either the hardware or the software. Prior flight as part of the pre-flight preparation, all embedded subsystems are tested and the internal bus communication is also validated. 
The current flight model is based on an Arduino compatible Atmega328p microntroller which combined with the Arduino IDE provides relatively easy development of the OBC. This design makes possible to use off the shelf Arduino boards during early development stage as breadboard models. Since the Arduino environment is easy to use it provides a fast learning curve for students in the beginning of their academic life.

\section{TELECOMMUNICATION (COM)}

The hardest and most vital part of a balloon mission is providing reliable 'telemetry and telecommand' (TC/TM) function. The UPRA platform provides two way radio communication to transmit telemetry and housekeeping to the ground (telemetry) and to receive control commands from the operators (telecommand). Students joining the telecommunication subsystem development team can learn the basics of radio communication and the design principals of high-frequency circuits.

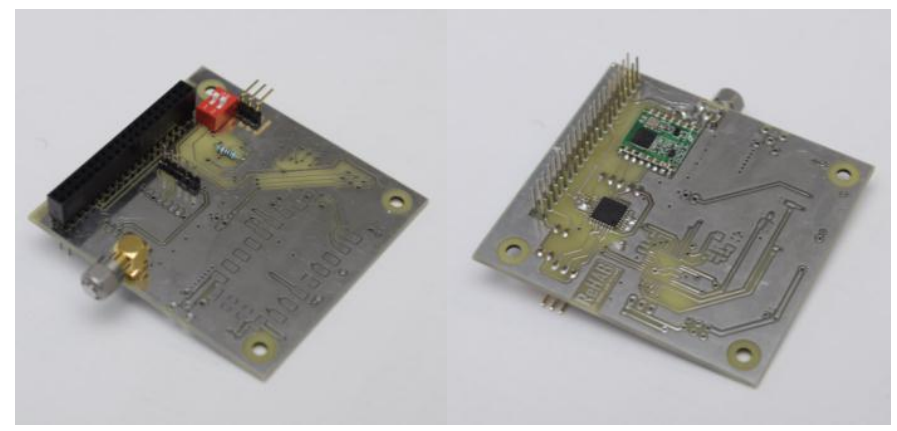

Fig. 3. UHF Communication subsystem with HOPE RF transceiver module

The current flying version of the communication subsystem is using an off-the shelf HOPE RF transceiver module (Fig. 3) which has limited efficiency. Students taking part in the further development of the COM can learn how to improve radio frequency $(\mathrm{RF})$ circuits, designing better filters and amplifiers and fine tuning high-frequency hardware elements. The goal is to change the factory assembled HOPE RF modules to a more stable custom made RF circuit developed by the UPRA Team.

\section{GROUND SEGMENT}

Alongside the on-board communication system, the ground segment is also under development. Currently the ground station transceiver hardware is the same as used on board the balloon platform. However it is equipped with a different firmware. This makes the ground connection highly compatible with the flying segment but provides very low configurability. As a preparation for later missions the upgrade process of the ground station is based on software defined radio (SDR) solutions. As part of the ground station development students will install an automated antenna rotator on the top of Schönherz Dormitory which is a nineteen story height building and the headquarters of our operations.

The ground station also needs a mission control software. Students interested in computer science can join the ground software development. The mission control software is a web based application using Python as backend and JavaScript as frontend. The goal is to develop a modular application which can be easily configured for different missions and different platforms. The current version of the software has already been supported several balloon flights and has been used to control a mobile rover at competitions.

Further development on the ground station software is to integrate with a live balloon trajectory predictor using live meteorological data thus providing real-time predictions on the landing site. Another goal is to use multiple radio stations simultaneously which could provide large coverage on balloon flights and can be the foundation of an advanced satellite tracking system.

\section{POWER SUPPLY AND DISTRIBUTION (EPS)}

The basic of all electrical equipment is analog circuitry. Students working on the Power Supply and Distribution subsystem can master their skills in analog electrical design and testing. This sub-system is a crucial part of any space- or near-spacecraft. Precise calculations, simulations and analysis should be performed in early development phase. However besides working on paper, students can gain experience in manufacturing, hand soldering, testing and fine tuning analog circuitry during the assembly, integration and verification (AIV) process.

Students interested in analog electronics can also work with different sensors and signal conditioning. On-board a spacecraft various analog sensors are used, usually connected to an analog to digital converter (ADC). Students working on sensor signal conditioning can learn how to amplify, filter or trace the given signal in longer distance. With these skills team members can aid the circuit design and development in any subsystem containing analog circuitry.

The Power Supply and Distribution sub-system and analog signal conditioning are the backbone of every space- and nearspacecraft. Students possessing knowledge in analog circuit design and experience in analog AIV will have advantage building their carrier in space industry.

\section{STRUCTURE AND INSULATION}

Mechanical structure is important to fly experiments to the stratosphere. Parts should withstand vibration, extreme temperature changes and mechanical impact. The $-60^{\circ} \mathrm{C}$ temperature in the stratosphere can be dangerous to the onboard electronics. COTS components used in the flight computer usually graded to $-40^{\circ} \mathrm{C}$ as minimum operating temperature. To provide reliable operation sufficient insulation needs to be applied around the electronic subsystems.

Designing the mechanical structure and insulation can help students to gain experience in Computer Aided Design (CAD), set up and run thermal and mechanical simulations using the CAD models of the payload train and learn the basics of production and pre-production activities. Our workshop is equipped with a $3 \mathrm{D}$ printing and a CNC milling facility which can provide hands-on experience in the production of mechanical elements. Our headquarters also has a small thermal cooling chamber which could provide $-16^{\circ} \mathrm{C}$ 
temperature. The cooling chamber can be used for preliminary testing and training workshops for students interested in testing and validation.

Since the launch of third party payloads started, a new challenge appeared in structure and insulation management. Equipment provided by customers requires a payload gondola. Currently the development of a general payload gondola is in progress which could provide standardized space for science payload on board of the UPRA platform. While most of the demands could fulfill with the standardized gondola there are payloads which requires custom made insulation and support structure (Fig. 4). The mechanical team should be able to design, manufacture and test these structural elements in a short deadline. This activity could be aided with the aforementioned $3 \mathrm{D}$ printer and advanced rapid prototyping techniques.

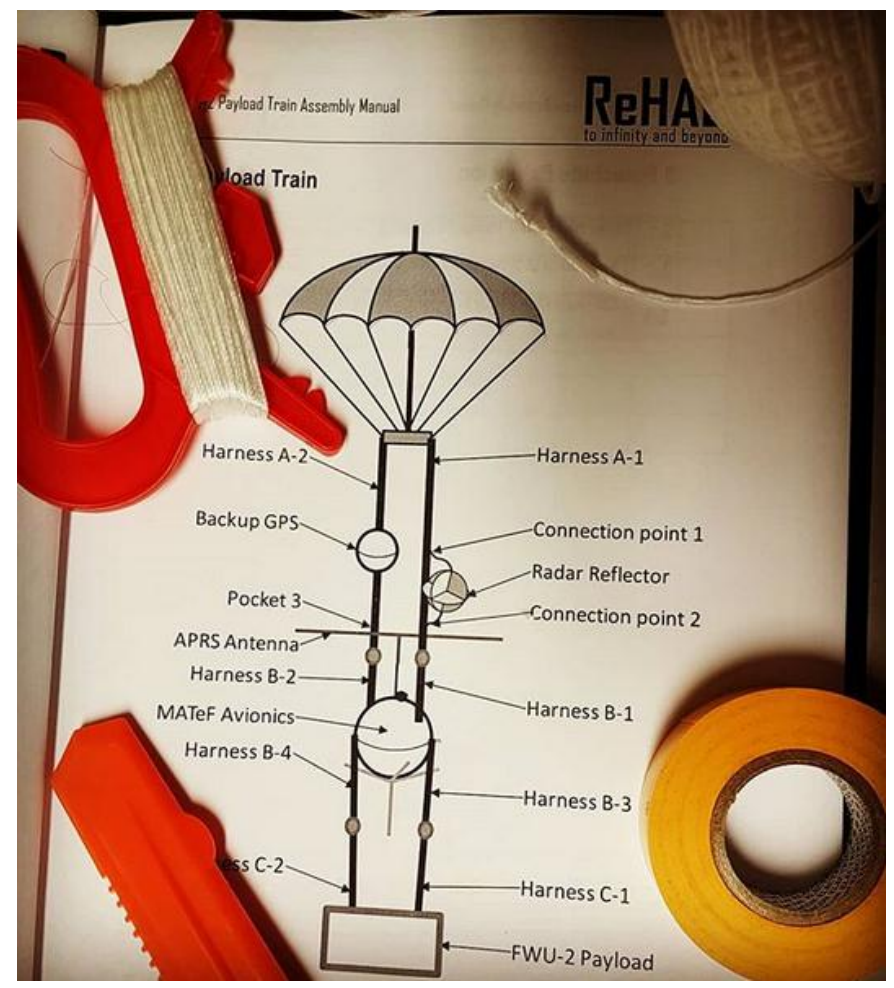

Fig. 4. AIV plan for the payload train with custom payload gondola

\section{BACKUP}

In space engineering fault-tolerant hardware must be developed due to the harsh environment and lack of maintainability. Reliability can be achieved through the validation process (Fig. 5) but testing is not always enough. In a spacecraft crucial subsystems usually have backups in a form of a hot- or cold redundant pair. The key objective during a high altitude balloon flight is to recover the payload after landing. The UPRA platform is designed with several backup tracking capabilities to ensure the reliability of the recovery process. Besides the main GPS - which is connected to the OnBoard Computer, - two independent GSM based trackers are part of the payload train. They are used to narrow the area of the landing site and act as a backup if the main on-board electronics fails.

The Telecommunication subsystem is also doubled. The flight computer uses the main GPS data to generate telemetry messages which then transmitted to the ground station via the main radio transceiver and as its hot redundant backup pair. The backup transmitter is only providing position data, but the message format used in the downlink does not requires a dedicated ground station, it could be decoded by automated HAM radio stations around the world.

All students work on this project have to learn the importance of fault-tolerant design and development to build reliable flight hardware. Using fault-analysis, verification and validation through the development process or gaining experience in redundant hardware design would give the students a point of view which can be advantageous in pursuing their space career.

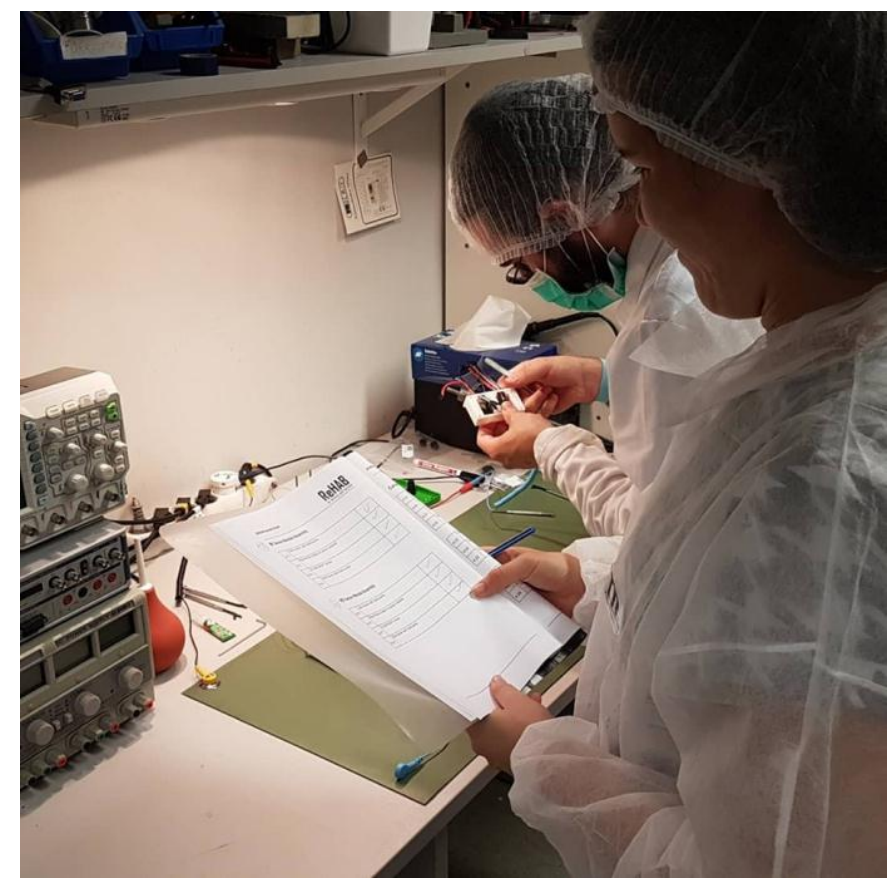

Fig. 5. Working on flying hardwer in 'grey' lab area to prevent contamination and ensure high quailty

\section{Flight Management}

In the case of a high altitude balloon without active control planning a precise flight path is not possible. Flight planning usually relies on predictions calculated by the physical properties of the payload train and meteorological data. To eliminate dangerous situations, students involved in flight planning activities should learn how to analyze meteorological data, calculate and use payload train parameters and update predictions using already available applications or develop new ones. Flight planning also includes the scheduling of the flight, communication with authorities, organize the launch and recovery team. During the flight the launch and recovery teams are supervised from the mission control and reliy on the flight plan. 
Launch events can be followed by civilian audience at the launch location aided with crowd control (Fig. 6). After the launch team secured the area they start the preparation of the launch equipment and the payload. The complete launch process is controlled by the launch director who follows a checklist that contains instruction on balloon inflation, platform and payload start up and GO-NOGO conditions. The launch director is in live contact with the flight director and ground station personnel to gather information on current mission status and report any malfunction during the launch process.

The recovery is organized in advance using the flight prediction data. The recovery team departs after launch and heads to the predicted landing area while maintaining continuous communication with the mission control and with the balloon. During the balloon retrieval the route of the recovery team regularly updated using newest available predictions and live telemetry data. After the successful recovery of the returning payload train it is the responsibility of the recovery team to properly shut down the flight system and ensure the safe transportation of the hardware.

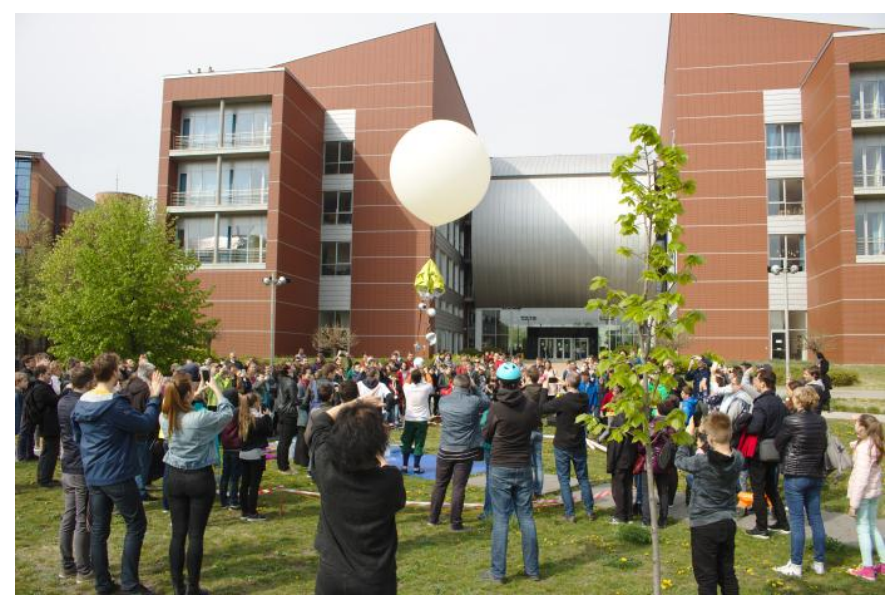

Fig. 6. Public balloon launch as part of Budapest Space Week with the audience of 'Kids University'

Launch and recovery team members can gain field experience during their activity. Being part of a field team needs great problem solving skills, proactive behavior and teamwork. These skills are important part in many fields of space engineering and can be beneficial for students seeking their carrier in space industry.

\section{SUMMARY}

Academic studies can be aided with projects providing hands-on experience to deepen the knowledge and further the horizon of students in their selected field. Students interested in space can have an opportunity to start their path by joining a high altitude balloon project. Working on near space equipment would help them gain the skillset needed for the space industry.

Since the beginning of the UPRA balloon project more than thirty BSc., ten MSc. and two PhD. students took part in the development. Most of them were studying electrical engineering and computer science. During the project timeline two thesis works have been accepted by the university. As the indicator of the project success we can proudly say that five of our alumni members have secured work opportunities in the space sector.

Our experience is that students who participated in the project had positive effects later on their professional carrier. The knowledge they gained during their time working with high altitude balloons is beneficial in many industries including fields of automotive, medical or aerospace engineering.

\section{REFERENCES}

[1] Gábor Fuszek, blikk.hu, 2019.

[2] Shane L. Larson et. al. - The first frontier: High altitude ballooning as a platform for student research experiences in science and engineering

[3] Jeremy Straub et. al. - A Design for Inspiring Students with Near-Space Exploration

[4] Zs.Bodó, B.Góczán - Educational Aspects of Developing a High Altitude Balloon Platform, H-Space Conference, 2019 